\title{
Weighted Composition Operators and Integral-Type Operators between Weighted Hardy Spaces on the Unit Ball
}

\author{
Stevo Stević ${ }^{\mathbf{1}}$ and Sei-Ichiro Ueki ${ }^{2}$ \\ ${ }^{1}$ Mathematical Institute of the Serbian Academy of Sciences, Knez Mihailova 36/III, 11000 Beograd, Serbia \\ ${ }^{2}$ Faculty of Engineering, Ibaraki University, Hitachi 316-8511, Japan \\ Correspondence should be addressed to Stevo Stević, sstevic@ptt.rs \\ Received 2 May 2009; Revised 3 June 2009; Accepted 8 June 2009 \\ Recommended by Leonid Berezansky \\ We study the boundedness and compactness of the weighted composition operators as well as \\ integral-type operators between weighted Hardy spaces on the unit ball. \\ Copyright (C) 2009 S. Stević and S.-I. Ueki. This is an open access article distributed under the \\ Creative Commons Attribution License, which permits unrestricted use, distribution, and \\ reproduction in any medium, provided the original work is properly cited.
}

\section{Introduction}

Let $\mathbb{B}$ denote the open unit ball of the $n$-dimensional complex vector space $\mathbb{C}^{n}, \partial \mathbb{B}$ its boundary, and let $H(\mathbb{B})$ denote the space of all holomorphic functions on $\mathbb{B}$. For $0<p<\infty$ and $\alpha \geq 0$ we define the weighted Hardy space $H_{\alpha}^{p}(\mathbb{B})$ as follows:

$$
H_{\alpha}^{p}(\mathbb{B})=\left\{f \in H(\mathbb{B}): \sup _{0<r<1}(1-r)^{\alpha} \int_{\partial \mathbb{B}}|f(r \zeta)|^{p} d \sigma(\zeta)<\infty\right\},
$$

where $d \sigma$ is the normalized Lebesgue measure on $\partial \mathbb{B}$ (see, also [1], as well as [2], for an equivalent definition of the space). Note that for $\alpha=0$ the weighted Hardy space becomes the Hardy space $H^{p}(\mathbb{B})$. We define the norm $\|\cdot\|_{H_{\alpha}^{p}}$ on this space as follows:

$$
\|f\|_{H_{\alpha}^{p}}^{p}=\sup _{0<r<1}(1-r)^{\alpha} \int_{\partial \mathbb{B}}|f(r \zeta)|^{p} d \sigma(\zeta)
$$


With this norm $H_{\alpha}^{p}(\mathbb{B})$ is a Banach space when $1 \leq p<\infty$. For a related space on the unit polydisk; see [3]. In this paper, we investigate two types of operators acting between weighted Hardy spaces.

Let $\varphi$ be a holomorphic self-map of $\mathbb{B}$ and $u \in H(\mathbb{B})$. Then $\varphi$ and $u$ induce a weighted composition operator $u C_{\varphi}$ on $H(\mathbb{B})$ which is defined by $u C_{\varphi} f=u(f \circ \varphi)$. This type of operators has been studied on various spaces of holomorphic functions in $\mathbb{C}^{n}$, by many authors; see, for example, [4], recent papers [5-17], and the references therein.

Let $g \in H(\mathbb{D})$ and $\varphi$ be a holomorphic self-map of the open unit disk $\mathbb{D}$ in the complex plane. Products of integral and composition operators on $H(\mathbb{D})$ were introduced by S. Li and S. Stević in a private communication (see [18-21], as well as papers [22] and [23] for closely related operators) as follows:

$$
\begin{aligned}
& C_{\varphi} J_{g} f(z)=\int_{0}^{\varphi(z)} f(\zeta) g^{\prime}(\zeta) d \zeta \\
& J_{g} C_{\varphi} f(z)=\int_{0}^{z} f(\varphi(\zeta)) g^{\prime}(\zeta) d \zeta .
\end{aligned}
$$

In [24] the first author of this paper has extended the operator in (1.4) in the unit ball settings as follows (see also $[25,26]$ ). Assume $g \in H(\mathbb{B}), g(0)=0$, and $\varphi$ is a holomorphic self-map of $\mathbb{B}$, then we define an operator on the unit ball as follows:

$$
P_{\varphi}^{g}(f)(z)=\int_{0}^{1} f(\varphi(t z)) g(t z) \frac{d t}{t}, \quad f \in H(\mathbb{B}), \quad z \in \mathbb{B} .
$$

If $n=1$, then $g \in H(\mathbb{D})$ and $g(0)=0$, so that $g(z)=z g_{0}(z)$, for some $g_{0} \in H(\mathbb{D})$. By the change of variable $\zeta=t z$, it follows that

$$
P_{\varphi}^{g} f(z)=\int_{0}^{1} f(\varphi(t z)) t z g_{0}(t z) \frac{d t}{t}=\int_{0}^{z} f(\varphi(\zeta)) g_{0}(\zeta) d \zeta .
$$

Thus the operator (1.5) is a natural extension of operator $J_{g} C_{\varphi}$ in (1.4). For related operators see [27-33] as well as the references therein.

In this paper we study the boundedness and compactness of the weighted composition operators as well as the integral-type operator $P_{\varphi}^{g}$, between different weighted Hardy spaces on the unit ball.

Throughout this paper, constants are denoted by $C$, they are positive and may differ from one occurrence to the other. The notation $a \leq b$ means that there is a positive constant $C$ such that $a \leq C b$. Moreover, if both $a \leq b$ and $b \leq a$ hold, then one says that $a \asymp b$.

\section{Weighted Composition Operators}

This section is devoted to studying weighted composition operators between weighted Hardy spaces. Weighted composition operators between different Hardy spaces on the unit ball were previously studied in $[15,34]$, while the composition operators on the unit ball were studied in $[35,36]$. For the case of the unit disk see also [37]. 
Before we formulate the main results in this section we quote several auxiliary results which will be used in the proofs of these ones.

Lemma 2.1. Let $0<p<\infty$ and $\alpha \geq 0$. Suppose that $u \in H(\mathbb{B})$ and $\varphi$ is a holomorphic self-map of $\mathbb{B}$. Then for each $f \in H(\mathbb{B})$

$$
\left\|u C_{\varphi} f\right\|_{H_{\alpha}^{p}} \leq \liminf _{R \rightarrow 1^{-}}\left\|u C_{\varphi} f_{R}\right\|_{H_{\alpha}^{p}}
$$

where $u C_{\varphi} f_{R}(z)=u(z) f(R \varphi(z))$.

Proof. Fix $r \in(0,1)$. Fatou's lemma shows that

$$
\begin{aligned}
(1-r)^{\alpha} \int_{\partial \mathbb{B}}|u(r \zeta) f(\varphi(r \zeta))|^{p} d \sigma(\zeta) & \leq(1-r)^{\alpha} \liminf _{R \rightarrow 1^{-}} \int_{\partial \mathbb{B}}|u(r \zeta) f(R \varphi(r \zeta))|^{p} d \sigma(\zeta) \\
& =\liminf _{R \rightarrow 1^{-}}(1-r)^{\alpha} \int_{\partial \mathbb{B}}|u(r \zeta) f(R \varphi(r \zeta))|^{p} d \sigma(\zeta) \\
& \leq \liminf _{R \rightarrow 1^{-}}\left\|u C_{\varphi} f_{R}\right\|_{H_{\alpha}^{p}}
\end{aligned}
$$

Hence we have the desired inequality.

Recall that an $f \in H(\mathbb{B})$ has the homogeneous expansion

$$
f(z)=\sum_{k=0}^{\infty} \sum_{|\gamma|=k} c(\gamma) z^{\gamma}
$$

where $\gamma=\left(\gamma_{1}, \ldots, \gamma_{n}\right)$ is a multi-index, $|\gamma|=\gamma_{1}+\cdots+\gamma_{n}$ and $z^{\gamma}=z_{1} \gamma_{1} \cdots z_{n}{ }^{\gamma_{n}}$. For the homogeneous expansion of $f$ and any integer $j \geq 1$, let

$$
R_{j} f(z)=\sum_{k=0}^{\infty} \sum_{|\gamma|=k} c(\gamma) z^{\gamma},
$$

and $K_{j}=I-R_{j}$ where $I f=f$ is the identity operator. Note that $K_{j}$ is compact operator on $H_{\alpha}^{p}(\mathbb{B})$ for each $j \in \mathbb{N}$.

Lemma 2.2. If $1<p<\infty$, then $R_{j}$ converges to 0 pointwise in the Hardy space $H^{p}(\mathbb{B})$ as $j \rightarrow \infty$.

Proof. See [34, Corollary 3.4] .

Lemma 2.2 and the uniform boundedness principle show that $\left\{R_{j}\right\}$ is an uniformly bounded sequence in $H^{p}(\mathbb{B})$. 
The following lemma is proved similar to [4, Lemma 3.16]. We omit its proof.

Lemma 2.3. If $u C_{\varphi}$ is bounded from $H_{\alpha}^{p}(\mathbb{B})$ into $H_{\beta}^{q}(\mathbb{B})$, then

$$
\left\|u C_{\varphi}\right\|_{e, H_{\alpha}^{p}(\mathbb{B}) \rightarrow H_{\beta}^{q}(\mathbb{B})} \leq \liminf _{j \rightarrow \infty}\left\|u C_{\varphi} R_{j}\right\|_{H_{\alpha}^{p}(\mathbb{B}) \rightarrow H_{\beta}^{q}(\mathbb{B})^{\prime}}
$$

where $\|\cdot\|_{e, H_{\alpha}^{p}(\mathbb{B}) \rightarrow H_{\beta}^{q}(\mathbb{B})}$ and $\|\cdot\|_{H_{\alpha}^{p}(\mathbb{B}) \rightarrow H_{\beta}^{q}(\mathbb{B})}$ denote the essential norm and the operator norm, respectively.

Lemma 2.4. Let $0<p \leq q<\infty$. Suppose that $\mu$ is a positive Borel measure on $\mathbb{B}$ which satisfies

$$
\mu(B(\zeta, t)) \leq C_{1} t^{q n / p} \quad(\zeta \in \partial \mathbb{B}, t>0)
$$

for some positive constant $C_{1}$. Then there exists a positive constant $C_{2}$ which depends only on $p, q$, and the dimension $n$ such that

$$
\int_{\mathbb{B}}|f|^{q} d \mu \leq C_{1} C_{2}\|f\|_{H^{p}}^{q}
$$

for any $f \in H^{p}(\mathbb{B})$. Here $B(\zeta, t)=\{z \in \mathbb{B}:|1-\langle z, \zeta\rangle|<t\}$.

Proof. See [38, page 13, Theorem ] or [34, Lemma 2.1] .

Let $0<q<\infty$. For each $r \in(0,1)$, a holomorphic self-map $\varphi$ of $\mathbb{B}$ and $u \in H(\mathbb{B})$, we define a positive Borel measure $\mu_{u, \varphi}^{r}$ on $\mathbb{B}$ by

$$
\mu_{u, \varphi}^{r}(E)=\int_{\left(\varphi_{r}\right)^{-1}(E)}\left|u_{r}\right|^{q} d \sigma
$$

for all Borel sets $E$ of $\mathbb{B}$. By the change of variables formula from measure theory, we can verify

$$
\int_{\mathbb{B}} g d \mu_{u, \varphi}^{r}=\int_{\partial \mathbb{B}}|u(r \zeta)|^{q}(g \circ \varphi)(r \zeta) d \sigma(\zeta),
$$

for each nonnegative measurable function $g$ in $\mathbb{B}$.

Theorem 2.5. Let $0<p \leq q<\infty$ and $\alpha, \beta \geq 0$. Suppose that $u \in H(\mathbb{B})$ and $\varphi$ is a holomorphic self-map of $\mathbb{B}$. Then $u C_{\varphi}: H_{\alpha}^{p}(\mathbb{B}) \rightarrow H_{\beta}^{q}(\mathbb{B})$ is bounded if and only if

$$
\sup _{w \in \mathbb{B}} \sup _{0<r<1}(1-r)^{\beta} \int_{\partial \mathbb{B}}|u(r \zeta)|^{q}\left\{\frac{1-|w|^{2}}{|1-\langle\varphi(r \zeta), w\rangle|^{2}}\right\}^{q(\alpha+n) / p} d \sigma(\zeta)<\infty .
$$


Proof. For $w \in \mathbb{B}$ we put

$$
f_{w}(z)=\left\{\frac{1-|w|^{2}}{(1-\langle z, w\rangle)^{2}}\right\}^{(\alpha+n) / p}
$$

Then we see that $f_{w} \in H_{\alpha}^{p}(\mathbb{B})$ and moreover $\sup _{w \in \mathbb{B}}\left\|f_{w}\right\|_{H_{\alpha}^{p}} \leq C$. By a straightforward calculation, we have

$$
\left\|u C_{\varphi} f_{w}\right\|_{H_{\beta}^{q}}^{q}=\sup _{0<r<1}(1-r)^{\beta} \int_{\partial \mathbb{B}}|u(r \zeta)|^{q}\left\{\frac{1-|w|^{2}}{|1-\langle\varphi(r \zeta), w\rangle|^{2}}\right\}^{q(\alpha+n) / p} d \sigma(\zeta),
$$

for all $w \in \mathbb{B}$. Hence if $u C_{\varphi}: H_{\alpha}^{p}(\mathbb{B}) \rightarrow H_{\beta}^{q}(\mathbb{B})$ is bounded, then $u$ and $\varphi$ satisfy the condition

$$
\sup _{w \in \mathbb{B} 0<r<1}(1-r)^{\beta} \int_{\partial \mathbb{B}}|u(r \zeta)|^{q}\left\{\frac{1-|w|^{2}}{|1-\langle\varphi(r \zeta), w\rangle|^{2}}\right\}^{q(\alpha+n) / p} d \sigma(\zeta) \leq C\left\|u C_{\varphi}\right\|_{H_{\alpha}^{p}(\mathbb{B}) \rightarrow H_{\beta}^{q}(\mathbb{B})}^{q}<\infty .
$$

Next we assume

$$
M:=\sup _{w \in \mathbb{B}} \sup _{0<r<1}(1-r)^{\beta} \int_{\partial \mathbb{B}}|u(r \zeta)|^{q}\left\{\frac{1-|w|^{2}}{|1-\langle\varphi(r \zeta), w\rangle|^{2}}\right\}^{q(\alpha+n) / p} d \sigma(\zeta)<\infty .
$$

Fix $r \in(0,1)$ and $R \in(0,1)$, respectively. For $\zeta \in \partial \mathbb{B}$ and $t, 0<t \leq t_{R}=1-R$, we put $w=(1-t) \zeta$ and $w_{R}=\left(1-t_{R}\right) \zeta$. Since the function $f_{w}(z)$, which is defined by $(2.11)$ for this $w$, satisfies

$$
\left|f_{w}(z)\right|^{q}>4^{-q(\alpha+n) / p} t^{-q n / p}(1-R)^{-q \alpha / p}
$$

for all $z \in B(\zeta, t)$, we have

$$
\begin{aligned}
\frac{\mu_{u, \varphi}^{r}(B(\zeta, t))}{t^{q n / p}} & \leq 4^{q(\alpha+n) / p}(1-R)^{q \alpha / p} \int_{B(\zeta, t)}\left|f_{w}(z)\right|^{q} d \mu_{u, \varphi}^{r}(z) \\
& \leq 4^{q(\alpha+n) / p}(1-R)^{q \alpha / p} \int_{\partial \mathbb{B}}|u(r \zeta)|^{q}\left\{\frac{1-|w|^{2}}{|1-\langle\varphi(r \zeta), w\rangle|^{2}}\right\}^{q(\alpha+n) / p} d \sigma(\zeta) \\
& \leq 4^{q(\alpha+n) / p} \frac{(1-R)^{q \alpha / p}}{(1-r)^{\beta}} M
\end{aligned}
$$

By the same argument, the function $f_{w_{R}}(z)$ gives the following estimate:

$$
\mu_{u, \varphi}^{r}\left(B\left(\zeta, 2 t_{R}\right)\right) \leq 9^{q(\alpha+n) / p} \frac{(1-R)^{q \alpha / p}}{(1-r)^{\beta}} M t_{R}^{q n / p}
$$


Now we need to prove that there exists a positive constant $C$ such that

$$
\mu_{u, \varphi}^{r}(B(\zeta, t)) \leq C \frac{(1-R)^{q \alpha / p}}{(1-r)^{\beta}} M t^{q n / p}
$$

for all $\zeta \in \partial \mathbb{B}$ and $t>0$. By the estimate (2.16), we see that the inequality (2.18) is true for all $t \in\left(0, t_{R}\right]$. Thus we assume $t>t_{R}$. By the same argument as in [36, pages 241-242, proof of Theorem 1.1], we see that the inequality (2.17) shows that there exists a positive constant $C_{n}$ which depends only on the dimension $n$ such that

$$
\begin{aligned}
\mu_{u, \varphi}^{r}(B(\zeta, t)) & \leq C_{n}\left(\frac{t}{t_{R}}\right)^{n} 9^{q(\alpha+n) / p} \frac{(1-R)^{q \alpha / p}}{(1-r)^{\beta}} M t_{R}^{q n / p} \\
& =C_{n} 9^{q(\alpha+n) / p} \frac{(1-R)^{q \alpha / p}}{(1-r)^{\beta}} M t^{n} t_{R}^{(q / p-1) n} \\
& \leq C_{n} 9^{q(\alpha+n) / p} \frac{(1-R)^{q \alpha / p}}{(1-r)^{\beta}} M t^{q n / p} .
\end{aligned}
$$

Hence for $C=\max \left\{4^{q(\alpha+n) / p}, C_{n} 9^{q(\alpha+n) / p}\right\}$, we have the inequality in (2.18).

For $f \in H_{\alpha}^{p}(\mathbb{B})$ the dilate function $f_{R}$ belongs to the ball algebra, and so $f_{R}$ is in the Hardy space $H^{p}(\mathbb{B})$. Hence Lemma 2.4 gives

$$
\int_{\mathbb{B}}\left|f_{R}(z)\right|^{q} d \mu_{u, \varphi}^{r}(z) \leq C^{\prime} C \frac{(1-R)^{q \alpha / p}}{(1-r)^{\beta}} M\left\|f_{R}\right\|_{H^{p}}^{q}
$$

for some positive constant $C^{\prime}$ and all $R \in(0,1)$. This implies that

$$
(1-r)^{\beta} \int_{\partial \mathbb{B}}\left|u C_{\varphi} f_{R}(r \zeta)\right|^{q} d \sigma(\zeta) \leq C^{\prime} C M\left[(1-R)^{\alpha} \int_{\partial \mathbb{B}}|f(R \zeta)|^{p} d \sigma(\zeta)\right]^{q / p},
$$

and so we have

$$
\left\|u C_{\varphi} f_{R}\right\|_{H_{\beta}^{q}}^{q} \leq C^{\prime} C M\|f\|_{H_{\alpha}^{p}}^{q}
$$

for all $R \in(0,1)$. By Lemma 2.1 we have

$$
\begin{aligned}
\left\|u C_{\varphi} f\right\|_{H_{\beta}^{q}}^{q} \leq & C^{\prime} C\|f\|_{H_{\alpha}^{p}}^{q} \\
& \times \sup _{w \in \mathbb{B} 0<r<1}(1-r)^{\beta} \int_{\partial \mathbb{B}}|u(r \zeta)|^{q}\left\{\frac{1-|w|^{2}}{|1-\langle\varphi(r \zeta), w\rangle|^{2}}\right\}^{q(\alpha+n) / p} d \sigma(\zeta) .
\end{aligned}
$$

This completes the proof. 
The following proposition is proved in a standard way; see, for example, the proofs of the corresponding results in $[4,32,33,39]$. Hence we omit its proof.

Proposition 2.6. Let $0<p, q<\infty$ and $\alpha, \beta \geq 0$. Suppose that $u \in H(\mathbb{B})$ and $\varphi$ is a holomorphic selfmap of $\mathbb{B}$ which induce the bounded operator $u C_{\varphi}: H_{\alpha}^{p}(\mathbb{B}) \rightarrow H_{\beta}^{q}(\mathbb{B})$. Then $u C_{\varphi}: H_{\alpha}^{p}(\mathbb{B}) \rightarrow H_{\beta}^{q}(\mathbb{B})$ is compact if and only if for every bounded sequence $\left\{f_{j}\right\}_{j \in \mathbb{N}}$ in $H_{\alpha}^{p}(\mathbb{B})$ which converges to 0 uniformly on compact subsets of $\mathbb{B},\left\{u C_{\varphi} f_{j}\right\}_{j \in \mathbb{N}}$ converges to 0 in $H_{\beta}^{q}(\mathbb{B})$.

In the proof of Theorem 2.8, we need the following lemma.

Lemma 2.7. Let $1<p<\infty, \alpha \geq 0$, and $f_{w}$ be the family of test functions defined in (2.11). Then $f_{w} \rightarrow 0$ weakly in $H_{\alpha}^{p}(\mathbb{B})$ as $|w| \rightarrow 1^{-}$.

Proof. The family $\left\{f_{w}\right\}_{w \in \mathbb{B}}$ is bounded in $H_{\alpha}^{p}(\mathbb{B})$ and $f_{w} \rightarrow 0$ uniformly on compact subsets of $\mathbb{B}$ as $|w| \rightarrow 1^{-}$. By the definitions of the space $H_{\alpha}^{p}(\mathbb{B})$ and the norm $\|\cdot\|_{H_{\alpha}^{p} \text {, we see that }}$ $H_{\alpha}^{p}(\mathbb{B})$ is a subspace of the weighted Bergman space $A_{\alpha}^{p}(\mathbb{B})$ and

$$
\|f\|_{A_{\alpha}^{p}} \leq C(\alpha, p, n)\|f\|_{H_{\alpha}^{p}}\left(f \in H_{\alpha}^{p}(\mathbb{B})\right)
$$

for some positive constant $C(\alpha, p, n)$ which depends on $\alpha, p$, and $n$. This inequality implies that the family $\left\{f_{w}\right\}_{w \in \mathbb{B}}$ is also bounded in $A_{\alpha}^{p}(\mathbb{B})$. Note also that the family converges to 0 uniformly on compact subsets of $\mathbb{B}$ as $|w| \rightarrow 1^{-}$. Hence $f_{w} \rightarrow 0$ weakly in $A_{\alpha}^{p}(\mathbb{B})$ as $|w| \rightarrow 1^{-}$.

In order to prove that $f_{w} \rightarrow 0$ weakly in $H_{\alpha}^{p}(\mathbb{B})$ as $|w| \rightarrow 1^{-}$, we take an arbitrary bounded linear functional $\Lambda$ on $H_{\alpha}^{p}(\mathbb{B})$. By the Hahn-Banach theorem, $\Lambda$ can be extended to a bounded linear functional $\tilde{\Lambda}$ on $A_{\alpha}^{p}(\mathbb{B})$ so that $\tilde{\Lambda}\left(f_{w}\right)=\Lambda\left(f_{w}\right)$ for all $w \in \mathbb{B}$. Since $f_{w} \rightarrow 0$ weakly in $A_{\alpha}^{p}(\mathbb{B})$ as $|w| \rightarrow 1^{-}$, we have $\Lambda\left(f_{w}\right)=\widetilde{\Lambda}\left(f_{w}\right) \rightarrow 0$ as $|w| \rightarrow 1^{-}$, and so $f_{w} \rightarrow 0$ weakly in $H_{\alpha}^{p}(\mathbb{B})$ as $|w| \rightarrow 1^{-}$.

Theorem 2.8. Let $1<p \leq q<\infty$ and $\alpha, \beta \geq 0$. Suppose that $u \in H(\mathbb{B})$ and $\varphi$ is a holomorphic self-map of $\mathbb{B}$ such that $u C_{\varphi}: H_{\alpha}^{p}(\mathbb{B}) \rightarrow H_{\beta}^{q}(\mathbb{B})$ is bounded. Then the qth power of the essential norm $\left\|u C_{\varphi}\right\|_{e, H_{\alpha}^{p}(\mathbb{B}) \rightarrow H_{\beta}^{q}(\mathbb{B})}$ is comparable to

$$
\limsup _{|w| \rightarrow 1^{-}} \sup _{0<r<1}(1-r)^{\beta} \int_{\partial \mathbb{B}}|u(r \zeta)|^{q}\left\{\frac{1-|w|^{2}}{|1-\langle\varphi(r \zeta), w\rangle|^{2}}\right\}^{q(\alpha+n) / p} d \sigma(\zeta) .
$$

Hence $u C_{\varphi}: H_{\alpha}^{p}(\mathbb{B}) \rightarrow H_{\beta}^{q}(\mathbb{B})$ is compact if and only if

$$
\lim _{|w| \rightarrow 1^{-}} \sup _{0<r<1}(1-r)^{\beta} \int_{\partial \mathbb{B}}|u(r \zeta)|^{q}\left\{\frac{1-|w|^{2}}{|1-\langle\varphi(r \zeta), w\rangle|^{2}}\right\}^{q(\alpha+n) / p} d \sigma(\zeta)=0 .
$$


Proof. To prove a lower estimate

$$
\left\|u C_{\varphi}\right\|_{e, H_{\alpha}^{p}(\mathbb{B}) \rightarrow H_{\beta}^{q}(\mathbb{B})}^{q} \geq \limsup _{|w| \rightarrow 1^{-}} \sup _{0<r<1}(1-r)^{\beta} \int_{\partial \mathbb{B}}|u(r \zeta)|^{q}\left\{\frac{1-|w|^{2}}{|1-\langle\varphi(r \zeta), w\rangle|^{2}}\right\}^{q(\alpha+n) / p} d \sigma(\zeta),
$$

we consider the test functions $f_{w}$ defined in (2.11). The family $\left\{f_{w}\right\}_{w \in \mathbb{B}}$ is bounded in $H_{\alpha}^{p}(\mathbb{B})$, say by $L$, and $f_{w} \rightarrow 0$ uniformly on compact subsets of $\mathbb{B}$ as $|w| \rightarrow 1^{-}$. Thus by Lemma 2.7 we have that $f_{w} \rightarrow 0$ weakly in $H_{\alpha}^{p}(\mathbb{B})$ as $|w| \rightarrow 1^{-}$, so that $\left\|\mathcal{K} f_{w}\right\|_{H_{\beta}^{q}} \rightarrow 0$ as $|w| \rightarrow 1^{-}$for every compact operator $\mathcal{K}: H_{\alpha}^{p}(\mathbb{B}) \rightarrow H_{\beta}^{q}(\mathbb{B})$. Hence

$$
\begin{aligned}
L\left\|u C_{\varphi}-\mathcal{K}\right\|_{H_{\alpha}^{p}(\mathbb{B}) \rightarrow H_{\beta}^{q}(\mathbb{B})} & \geq \limsup _{|w| \rightarrow 1^{-}}\left\|\left(u C_{\varphi}-\mathcal{K}\right) f_{w}\right\|_{H_{\beta}^{q}} \\
& \geq \limsup _{|w| \rightarrow 1^{-}}\left\|u C_{\varphi} f_{w}\right\|_{H_{\beta}^{q}} .
\end{aligned}
$$

This inequality and (2.12) give the lower estimate for $\left\|u C_{\varphi}\right\|_{e, H_{\alpha}^{p}(\mathbb{B}) \rightarrow H_{\beta}^{q}(\mathbb{B})}^{q}$

Next we prove an upper estimate. Take $f \in H_{\alpha}^{p}(\mathbb{B})$ with $\|f\|_{H_{\alpha}^{p}} \leq 1$. Fix $\varepsilon>0$ and put

$$
M_{1}:=\limsup _{|w| \rightarrow 1^{-}} \sup _{0<r<1}(1-r)^{\beta} \int_{\partial \mathbb{B}}|u(r \zeta)|^{q}\left\{\frac{1-|w|^{2}}{|1-\langle\varphi(r \zeta), w\rangle|^{2}}\right\}^{q(\alpha+n) / p} d \sigma(\zeta)
$$

Then we can choose $R_{0} \in(0,1)$ such that

$$
\sup _{0<r<1}(1-r)^{\beta} \int_{\partial \mathbb{B}}|u(r \zeta)|^{q}\left\{\frac{1-|w|^{2}}{|1-\langle\varphi(r \zeta), w\rangle|^{2}}\right\}^{q(\alpha+n) / p} d \sigma(\zeta)<M_{1}+\varepsilon,
$$

for $w \in \mathbb{B}$ with $|w|>R_{0}$. Fix $r \in(0,1)$ and $R \in\left(R_{0}, 1\right)$. By the same argument as in the proof of inequality (2.20) in Theorem 2.5, we obtain that

$$
\int_{\mathbb{B}}\left|\left(R_{j} f\right)_{R}(z)\right|^{q} d \mu_{u, \varphi}^{r}(z) \leq C \frac{(1-R)^{q \alpha / p}}{(1-r)^{\beta}}\left(M_{1}+\varepsilon\right)\left\|\left(R_{j} f\right)_{R}\right\|_{H^{p}}^{q}
$$

where the positive constant $C$ is independent of $r, R$ and a positive integer $j$. Since $f_{R}$ is in the ball algebra, Lemma 2.2 gives

$$
\left\|\left(R_{j} f\right)_{R}\right\|_{H^{p}}^{q}=\left\|R_{j}\left(f_{R}\right)\right\|_{H^{p}}^{q} \leq \sup _{j \geq 1}\left\|R_{j}\right\|_{H^{p}(\mathbb{B}) \rightarrow H^{p}(\mathbb{B})}^{q}\left\|f_{R}\right\|_{H^{p}}^{q}
$$


Combining this with (2.31), we have

$$
\begin{aligned}
(1-r)^{\beta} \int_{\partial \mathbb{B}}\left|u C_{\varphi}\left(R_{j} f_{R}\right)(r \zeta)\right|^{q} d \sigma(\zeta) & \leq C^{\prime}\left(M_{1}+\varepsilon\right)\left[(1-R)^{\alpha} \int_{\partial \mathbb{B}}\left|f_{R}(\zeta)\right|^{p} d \sigma(\zeta)\right]^{q / p} \\
& \leq C^{\prime}\left(M_{1}+\varepsilon\right)\|f\|_{H_{\alpha}^{p},}^{q}
\end{aligned}
$$

and so we have

$$
\left\|u C_{\varphi}\left(R_{j} f_{R}\right)\right\|_{H_{\beta}^{q}}^{q} \leq C^{\prime}\left(M_{1}+\varepsilon\right)\|f\|_{H_{\alpha}^{p}}^{q}
$$

Letting $R \rightarrow 1^{-}$, by Lemma 2.1, we obtain

$$
\left\|u C_{\varphi} R_{j} f\right\|_{H_{\beta}^{q}}^{q} \leq C^{\prime}\left(M_{1}+\varepsilon\right)\|f\|_{H_{\alpha}^{p}}^{q}
$$

Since $\varepsilon>0$ is arbitrary, this estimate and Lemma 2.3 imply

$$
\begin{aligned}
\left\|u C_{\varphi}\right\|_{e, H_{\alpha}^{p}(\mathbb{B}) \rightarrow H_{\beta}^{q}(\mathbb{B})}^{q} & \leq \liminf _{j \rightarrow \infty}\left\|u C_{\varphi} R_{j}\right\|_{H_{\alpha}^{p}(\mathbb{B}) \rightarrow H_{\beta}^{q}(\mathbb{B})}^{q} \\
& \leq C^{\prime} \limsup _{|w| \rightarrow 1^{-} 0<r<1}(1-r)^{\beta} \int_{\partial \mathbb{B}}|u(r \zeta)|^{q}\left\{\frac{1-|w|^{2}}{|1-\langle\varphi(r \zeta), w\rangle|^{2}}\right\}^{q(\alpha+n) / p} d \sigma(\zeta),
\end{aligned}
$$

which completes the proof.

Remark 2.9. In the above proof, we used Lemma 2.2. This lemma required the assumption $1<$ $p<\infty$. Hence we cannot have an upper estimate for $\left\|u C_{\varphi}\right\|_{e, H_{\alpha}^{p}(\mathbb{B}) \rightarrow H_{\beta}^{q}(\mathbb{B})}$ in the case $0<p \leq 1$. However, Proposition 2.6 shows that the compactness of $u C_{\varphi}: H_{\alpha}^{p}(\mathbb{B}) \rightarrow H_{\beta}^{q}(\mathbb{B})(0<p \leq q<$ $\infty)$ is equivalent to

$$
\lim _{|w| \rightarrow 1^{-}} \sup _{0<r<1}(1-r)^{\beta} \int_{\partial \mathbb{B}}|u(r \zeta)|^{q}\left\{\frac{1-|w|^{2}}{|1-\langle\varphi(r \zeta), w\rangle|^{2}}\right\}^{q(\alpha+n) / p} d \sigma(\zeta)=0
$$

\section{Integral-Type Operators}

Here we study the boundedness and compactness of the integral-type operators $P_{\varphi}^{g}$ between weighted Hardy spaces on the unit ball.

For $f \in H(\mathbb{B})$ with the Taylor expansion $f(z)=\sum_{|\gamma| \geq 0} a_{\gamma} z^{\gamma}$, let $\mathfrak{R} f(z)=\sum_{|\gamma| \geq 0}|\gamma| a_{\gamma} z^{\gamma}$ be the radial derivative of $f$. 
The following lemma was proved in [24] (see also [25]).

Lemma 3.1. Assume that $\varphi$ is a holomorphic self-map of $\mathbb{B}, g \in H(\mathbb{B})$ and $g(0)=0$. Then for every $f \in H(\mathbb{B})$ one holds

$$
\mathfrak{R}\left[P_{\varphi}^{g}(f)\right](z)=f(\varphi(z)) g(z) .
$$

A positive continuous function $\omega$ on the interval $[0,1)$ is called normal [40] if there is a $\delta \in[0,1)$ and $a$ and $b, 0<a<b$ such that

$$
\begin{aligned}
& \frac{\omega(r)}{(1-r)^{a}} \text { is decreasing on }[\delta, 1) \text { and } \lim _{r \rightarrow 1} \frac{\omega(r)}{(1-r)^{a}}=0, \\
& \frac{\omega(r)}{(1-r)^{b}} \text { is increasing on }[\delta, 1) \text { and } \lim _{r \rightarrow 1} \frac{\omega(r)}{(1-r)^{b}}=\infty .
\end{aligned}
$$

If it is said that a function $\omega: \mathbb{B} \rightarrow[0, \infty)$ is normal, it is also assume that it is radial.

Lemma 3.2. Assume that $0<q \leq \infty, m$ is a positive integer and $\omega$ is normal. Then for every $f \in H(\mathbb{B})$

$$
\sup _{0<r<1} \omega(r) M_{q}(f, r) \asymp|f(0)|+\sup _{0<r<1}(1-r)^{m} \omega(r) M_{q}\left(\Re^{m} f, r\right),
$$

where

$$
M_{q}(f, r)=\left(\int_{\partial \mathbb{B}}|f(r \zeta)|^{q} d \sigma(\zeta)\right)^{1 / q}, \quad M_{\infty}(f, r)=\sup _{\zeta \in \partial \mathbb{B}}|f(r \zeta)|
$$

Proof. The proof of the lemma in the case $1 \leq q \leq \infty$ can be found in [27, Theorem 2]. However, due to an overlook, the proof for the case $q \in(0,1)$ has a gap. Hence we will give a correct proof here in the case.

We may assume that $f(0)=0$, otherwise we can consider the functions $h(z)=f(z)-$ $f(0)$. Also we may assume that $\delta=0$, to avoid some minor technical difficulties.

By [27, Lemma 1$]$, for each fixed $q \in(0,1]$, there is a positive constant $C$ depending only on $q$ and the dimension $n$ such that

$$
M_{q}(f, r) \leq \frac{C}{r}\left(\int_{0}^{r}(r-t)^{q-1} M_{q}^{q}(\Re f, t) d t\right)^{1 / q}
$$

for every $r \in(0,1)$ and $f \in H(\mathbb{B})$ such that $f(0)=0$. 
From (3.5) and the fact that $\omega$ is normal, we have

$$
\begin{aligned}
\sup _{0 \leq r<1} \omega(r) M_{q}(f, r) & \leq C \sup _{0 \leq r<1} \omega(r) \frac{1}{r}\left(\int_{0}^{r}(r-t)^{q-1} M_{q}^{q}(\Re f, t) d t\right)^{1 / q} \\
& \leq C \sup _{0 \leq r<1}(1-r)^{a} \frac{1}{r}\left(\int_{0}^{r}(r-t)^{q-1} \frac{\omega^{q}(t)}{(1-t)^{a q}} M_{q}^{q}(\Re f, t) d t\right)^{1 / q} \\
& \leq C \sup _{0 \leq r<1}(1-r)^{a} \frac{1}{r}\left(\int_{0}^{r} \frac{(r-t)^{q-1}}{(1-t)^{q q+q}} d t\right)^{1 / q} \sup _{0 \leq t<1}(1-t) \omega(t) M_{q}(\Re f, t) \\
& =C \sup _{0 \leq r<1}(1-r)^{a}\left(\int_{0}^{1} \frac{(1-u)^{q-1}}{(1-u r)^{q q+q}} d u\right)^{1 / q} \sup _{0 \leq t<1}(1-t) \omega(t) M_{q}(\Re f, t) .
\end{aligned}
$$

By [40, page 291, Lemma 6] there exists a positive constant $C$ such that

$$
\int_{0}^{1} \frac{(1-u)^{q-1}}{(1-u r)^{a q+q}} d u \leq \frac{C}{(1-r)^{a q}}
$$

for every $r \in(0,1)$. Combining this with (3.6), we have

$$
\begin{aligned}
\sup _{0 \leq r<1} \omega(r) M_{q}(f, r) & \leq \sup _{0 \leq r<1}(1-r)^{a}\left(\frac{1}{(1-r)^{a q}}\right)^{1 / q} \sup _{0 \leq t<1}(1-t) \omega(t) M_{q}(\Re f, t) \\
& =C \sup _{0 \leq t<1}(1-t) \omega(t) M_{q}(\Re f, t) .
\end{aligned}
$$

The reverse inequality is proved by the following inequality:

$$
(1-r) M_{q}(\Re f, r) \leq C M_{q}\left(f, \frac{1+r}{2}\right)
$$

and the fact that $\omega(r) \asymp \omega(1+r / 2)$ for $\omega$ normal (see [27]). Hence, we obtain the result for the case $m=1$.

For $m \geq 2$ it should be only noticed that $(1-r)^{m} \omega(r)$ is still normal, that $\Re^{m} f(0)=0$ for every integer $m \geq 1$, and use the method of induction.

Theorem 3.3. Let $0<p \leq q<\infty$ and $\alpha, \beta>0$. Suppose that $g \in H(\mathbb{B})$ with $g(0)=0$ and $\varphi$ is $a$ holomorphic self-map of $\mathbb{B}$. Then $P_{\varphi}^{g}: H_{\alpha}^{p}(\mathbb{B}) \rightarrow H_{\beta}^{q}(\mathbb{B})$ is bounded if and only if

$$
\sup _{w \in \mathbb{B}} \sup _{0<r<1}(1-r)^{\beta+q} \int_{\partial \mathbb{B}}|g(r \zeta)|^{q}\left\{\frac{1-|w|^{2}}{|1-\langle\varphi(r \zeta), w\rangle|^{2}}\right\}^{q(\alpha+n) / p} d \sigma(\zeta)<\infty .
$$


Proof. Take $f \in H_{\alpha}^{p}(\mathbb{B})$ with $\|f\|_{H_{\alpha}^{p}} \leq 1$. Since the function $(1-r)^{\beta / q}$ for $\beta>0$ and $0<q<\infty$ is normal, Lemma 3.2 gives

$$
\sup _{0<r<1}(1-r)^{\beta / q} M_{q}\left(P_{\varphi}^{g} f, r\right) \asymp\left|P_{\varphi}^{g} f(0)\right|+\sup _{0<r<1}(1-r)^{(\beta / q)+1} M_{q}\left(\mathfrak{R}\left[P_{\varphi}^{g} f\right], r\right) .
$$

The assumption $g(0)=0$ implies $P_{\varphi}^{g} f(0)=0$, and Lemma 3.1 shows $\Re\left[P_{\varphi}^{g} f\right]=g C_{\varphi} f$. Hence we obtain

$$
\sup _{0<r<1}(1-r)^{\beta} M_{q}^{q}\left(P_{\varphi}^{g} f, r\right) \asymp \sup _{0<r<1}(1-r)^{\beta+q} M_{q}^{q}\left(g C_{\varphi} f, r\right),
$$

and so we obtain $\left\|P_{\varphi}^{g} f\right\|_{H_{\beta}^{q}}^{q} \asymp\left\|g C_{\varphi} f\right\|_{H_{\beta+q}^{q}}^{q}$. This implies that the boundedness of $P_{\varphi}^{g}: H_{\alpha}^{p}(\mathbb{B}) \rightarrow$ $H_{\beta}^{q}(\mathbb{B})$ is equivalent to the boundedness of $g C_{\varphi}: H_{\alpha}^{p}(\mathbb{B}) \rightarrow H_{\beta+q}^{q}(\mathbb{B})$. So Theorem 2.5 shows that the condition

$$
\sup _{w \in \mathbb{B}} \sup _{0<r<1}(1-r)^{\beta+q} \int_{\partial \mathbb{B}}|g(r \zeta)|^{q}\left\{\frac{1-|w|^{2}}{|1-\langle\varphi(r \zeta), w\rangle|^{2}}\right\}^{q(\alpha+n) / p} d \sigma(\zeta)<\infty
$$

is a necessary and sufficient condition for the boundedness of $P_{\varphi}^{g}: H_{\alpha}^{p}(\mathbb{B}) \rightarrow H_{\beta}^{q}(\mathbb{B})$. This completes the proof.

The next proposition is proved similar to Proposition 2.6.

Proposition 3.4. Let $0<p, q<\infty$, and $\alpha, \beta>0$. Suppose that $g \in H(\mathbb{B})$ with $g(0)=0$ and $\varphi$ is a holomorphic self-map of $\mathbb{B}$ which induce the bounded operator $P_{\varphi}^{g}: H_{\alpha}^{p}(\mathbb{B}) \rightarrow H_{\beta}^{q}(\mathbb{B})$. Then $P_{\varphi}^{g}: H_{\alpha}^{p}(\mathbb{B}) \rightarrow H_{\beta}^{q}(\mathbb{B})$ is compact if and only if for every bounded sequence $\left\{f_{j}\right\}_{j \in \mathbb{N}}$ in $H_{\alpha}^{p}(\mathbb{B})$ which converges to 0 uniformly on compact subsets of $\mathbb{B},\left\{P_{\varphi}^{g} f_{j}\right\}_{j \in \mathbb{N}}$ converges to 0 in $H_{\beta}^{q}(\mathbb{B})$.

Theorem 3.5. Let $0<p \leq q<\infty$ and $\alpha, \beta>0$. Suppose that $g \in H(\mathbb{B})$ with $g(0)=0$ and $\varphi$ is a holomorphic self-map of $\mathbb{B}$ which induce the bounded operator $P_{\varphi}^{g}: H_{\alpha}^{p}(\mathbb{B}) \rightarrow H_{\beta}^{q}(\mathbb{B})$. Then $P_{\varphi}^{g}: H_{\alpha}^{p}(\mathbb{B}) \rightarrow H_{\beta}^{q}(\mathbb{B})$ is compact if and only if

$$
\lim _{|w| \rightarrow 1^{-}} \sup _{0<r<1}(1-r)^{\beta+q} \int_{\partial \mathbb{B}}|g(r \zeta)|^{q}\left\{\frac{1-|w|^{2}}{|1-\langle\varphi(r \zeta), w\rangle|^{2}}\right\}^{q(\alpha+n) / p} d \sigma(\zeta)=0 .
$$

Proof. First we assume that condition (3.14) holds. Take a bounded sequence $\left\{f_{j}\right\}_{j \in \mathbb{N}} \subset H_{\alpha}^{p}(\mathbb{B})$ which converges to 0 uniformly on compact subsets of $\mathbb{B}$. Theorem 2.8 and the remark in Section 2 show that $g C_{\varphi}: H_{\alpha}^{p}(\mathbb{B}) \rightarrow H_{\beta+q}^{q}(\mathbb{B})$ is compact. Thus Proposition 2.6 implies that

$$
\lim _{j \rightarrow \infty}\left\|g C_{\varphi} f_{j}\right\|_{H_{\beta+q}^{q}}=0 .
$$


From (3.15) and since $\left\|P_{\varphi}^{g} f_{j}\right\|_{H_{\beta}^{q}}^{q} \asymp\left\|g C_{\varphi} f_{j}\right\|_{H_{\beta+q}^{q}}^{q}$, we have that $\left\|P_{\varphi}^{g} f_{j}\right\|_{H_{\beta}^{q}}^{q} \rightarrow 0$ as $j \rightarrow \infty$. By Proposition 3.4, we see that $P_{\varphi}^{g}: H_{\alpha}^{p}(\mathbb{B}) \rightarrow H_{\beta}^{q}(\mathbb{B})$ is compact.

To prove the necessity of the condition in (3.14), we consider the family of test functions $f_{w}$ which is defined by (2.11). Hence we have

$$
\begin{aligned}
\left\|P_{\varphi}^{g} f_{w}\right\|_{H_{\beta}^{q}}^{q} & \asymp\left\|g C_{\varphi} f_{w}\right\|_{H_{\beta+q}^{q}}^{q} \\
& =\sup _{0<r<1}(1-r)^{\beta+q} \int_{\partial \mathbb{B}}|g(r \zeta)|^{q}\left\{\frac{1-|w|^{2}}{|1-\langle\varphi(r \zeta), w\rangle|^{2}}\right\}^{q(\alpha+n) / p} d \sigma(\zeta),
\end{aligned}
$$

for all $w \in \mathbb{B}$. Since $\left\{f_{w}\right\}_{w \in \mathbb{B}}$ is a bounded sequence in $H_{\alpha}^{p}(\mathbb{B})$ and $f_{w} \rightarrow 0$ uniformly on compact subsets of $\mathbb{B}$ as $|w| \rightarrow 1^{-}$, the compactness of $P_{\varphi}^{g}$ and Proposition 3.4 show that $\left\|P_{\varphi}^{g} f_{w}\right\|_{H_{\beta}^{q}}^{q} \rightarrow 0$ as $|w| \rightarrow 1^{-}$. This fact along with (3.16) implies the condition in (3.14), finishing the proof of the theorem.

Theorem 3.6. Let $1<p \leq q<\infty$ and $\alpha, \beta>0$. Suppose that $g \in H(\mathbb{B})$ with $g(0)=0$ and $\varphi$ is $a$ holomorphic self-map of $\mathbb{B}$ which induce the bounded operator $P_{\varphi}^{g}: H_{\alpha}^{p}(\mathbb{B}) \rightarrow H_{\beta}^{q}(\mathbb{B})$. Then the qth power of the essential norm of $P_{\varphi}^{g}$ is comparable to

$$
\limsup _{|w| \rightarrow 1^{-}} \sup _{0<r<1}(1-r)^{\beta+q} \int_{\partial \mathbb{B}}|g(r \zeta)|^{q}\left\{\frac{1-|w|^{2}}{|1-\langle\varphi(r \zeta), w\rangle|^{2}}\right\}^{q(\alpha+n) / p} d \sigma(\zeta) .
$$

Proof. To prove a lower estimate, we take an arbitrary compact operator $\mathcal{K}: H_{\alpha}^{p}(\mathbb{B}) \rightarrow$ $H_{\beta}^{q}(\mathbb{B})$. Since Lemma 2.7 implies that the family of functions $f_{w}$ defined by (2.11) converges to 0 weakly in $H_{\alpha}^{p}(\mathbb{B})$ as $|w| \rightarrow 1^{-}$, we obtain

$$
C\left\|P_{\varphi}^{g}-\mathcal{K}\right\|_{H_{\alpha}^{p}(\mathbb{B}) \rightarrow H_{\beta}^{q}(\mathbb{B})} \geq \limsup _{|w| \rightarrow 1^{-}}\left(\left\|P_{\varphi}^{g} f_{w}\right\|_{H_{\beta}^{q}}-\left\|\mathcal{K} f_{w}\right\|_{H_{\beta}^{q}}\right) \geq \limsup _{|w| \rightarrow 1^{-}}\left\|P_{\varphi}^{g} f_{w}\right\|_{H_{\beta}^{q}}
$$

Combining this with (3.16), we have

$$
C\left\|P_{\varphi}^{g}\right\|_{e, H_{\alpha}^{p}(\mathbb{B}) \rightarrow H_{\beta}^{q}(\mathbb{B})}^{q} \geq \limsup _{|w| \rightarrow 1^{-}} \sup _{0<r<1}(1-r)^{\beta+q} \int_{\partial \mathbb{B}}|g(r \zeta)|^{q}\left\{\frac{1-|w|^{2}}{|1-\langle\varphi(r \zeta), w\rangle|^{2}}\right\}^{q(\alpha+n) / p} d \sigma(\zeta),
$$

which is a lower estimate.

By some modification of Lemma 2.3 and the application of Lemmas 3.1 and 3.2, we get

$$
\begin{aligned}
& \left\|P_{\varphi}^{g}\right\|_{e, H_{\alpha}^{p}(\mathbb{B}) \rightarrow H_{\beta}^{q}(\mathbb{B})}^{q} \leq \liminf _{j \rightarrow \infty} \sup _{\|f\|_{H_{\alpha}^{p} \leq 1}}\left\|P_{\varphi}^{g} R_{j} f\right\|_{H_{\beta}^{q}}^{q} \\
& \leq C \liminf _{j \rightarrow \infty} \sup _{\|f\|_{H_{\alpha}^{p} \leq 1}}\left\|g C_{\varphi} R_{j} f\right\|_{H_{\beta+q}^{q}}^{q} .
\end{aligned}
$$


As in the proof of Theorem 2.8, we obtain

$$
\begin{aligned}
\liminf _{j \rightarrow \infty} \sup _{\|f\|_{H_{\alpha}^{p}}^{p \leq 1}}\left\|g C_{\varphi} R_{j} f\right\|_{H_{\beta+q}^{q}}^{q} \leq & C \limsup _{|w| \rightarrow 1^{-}} \sup _{0<r<1}(1-r)^{\beta+q} \\
& \times \int_{\partial \mathbb{B}}|g(r \zeta)|^{q}\left\{\frac{1-|w|^{2}}{|1-\langle\varphi(r \zeta), w\rangle|^{2}}\right\}^{q(\alpha+n) / p} d \sigma(\zeta),
\end{aligned}
$$

and so we have an upper estimate for $\left\|P_{\varphi}^{g}\right\|_{e, H_{\alpha}^{p}(\mathbb{B}) \rightarrow H_{\beta}^{q}(\mathbb{B})}^{q}$

\section{The Case $P_{\varphi}^{g}: H_{\alpha}^{\infty}(\mathbb{B}) \rightarrow H_{\beta}^{\infty}(\mathbb{B})$}

When $p=\infty$ and $\alpha>0$, we define the weighted-type space $H_{\alpha}^{\infty}(\mathbb{B})$ as follows:

$$
H_{\alpha}^{\infty}(\mathbb{B})=\left\{f \in H(\mathbb{B}): \sup _{0<r<1}(1-r)^{\alpha} M_{\infty}(f, r)<\infty\right\} .
$$

It is easy to see that $f \in H_{\alpha}^{\infty}(\mathbb{B})$ if and only if $\sup _{z \in \mathbb{B}}(1-|z|)^{\alpha}|f(z)|<\infty$, so we define the norm $\|f\|_{H_{\alpha}^{\infty}}$ on $H_{\alpha}^{\infty}(\mathbb{B})$ by this supremum.

Furthermore we consider the subspace $H_{\alpha, 0}^{\infty}(\mathbb{B})$ defined by

$$
H_{\alpha, 0}^{\infty}(\mathbb{B})=\left\{f \in H(\mathbb{B}): \lim _{r \rightarrow 1^{-}}(1-r)^{\alpha} M_{\infty}(f, r)=0\right\}
$$

Theorem 4.1. Let $\alpha, \beta>0$. Suppose that $g \in H(\mathbb{B})$ with $g(0)=0$ and $\varphi$ is a holomorphic self-map of $\mathbb{B}$. Then $P_{\varphi}^{g}: H_{\alpha}^{\infty}(\mathbb{B}) \quad\left(\right.$ or $\left.H_{\alpha, 0}^{\infty}(\mathbb{B})\right) \rightarrow H_{\beta}^{\infty}(\mathbb{B})$ is bounded if and only if

$$
\sup _{z \in \mathbb{B}} \frac{(1-|z|)^{\beta+1}|g(z)|}{(1-|\varphi(z)|)^{\alpha}}<\infty
$$

In this case, the operator norm $\left\|P_{\varphi}^{g}\right\|_{H_{\alpha}^{\infty}(\mathbb{B}) \quad\left(\text { or } H_{\alpha, 0}^{\infty}(\mathbb{B})\right) \rightarrow H_{\beta}^{\infty}(\mathbb{B})}$ is comparable to the above supremum.

Proof. By the definition of the space $H_{\alpha}^{\infty}(\mathbb{B}), f \in H_{\alpha}^{\infty}(\mathbb{B})$ satisfies the growth condition

$$
|f(w)| \leq(1-|w|)^{-\alpha}\|f\|_{H_{\alpha}^{\infty}} \quad(w \in \mathbb{B}),
$$

so it follows from Lemma 3.1 and Lemma 3.2 that

$$
\left\|P_{\varphi}^{g} f\right\|_{H_{\beta}^{\infty}} \asymp \sup _{z \in \mathbb{B}}(1-|z|)^{\beta+1}\left|g C_{\varphi} f(z)\right| \leq\|f\|_{H_{\alpha}^{\infty}} \sup _{z \in \mathbb{B}} \frac{(1-|z|)^{\beta+1}|g(z)|}{(1-|\varphi(z)|)^{\alpha}}
$$

for every $f \in H_{\alpha}^{\infty}(\mathbb{B})$. 
Hence we obtain

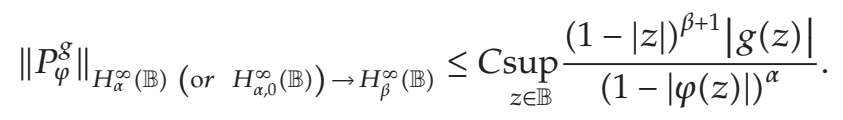

Now we prove the reverse inequality. For $w \in \mathbb{B}$, we put

$$
f_{w}(z)=\frac{1}{(1-\langle z, w\rangle)^{\alpha}} .
$$

Note that $f_{w} \in H_{\alpha, 0}^{\infty}(\mathbb{B})$ for each $w \in \mathbb{B}$ and moreover $\sup _{w \in \mathbb{B}}\left\|f_{w}\right\|_{H_{\alpha}^{\infty}} \leq 1$.

When $\varphi(z) \neq 0$, we have

$$
\begin{aligned}
\left\|P_{\varphi}^{g}\right\|_{H_{\alpha, 0}^{\infty}(\mathbb{B}) \rightarrow H_{\beta}^{\infty}(\mathbb{B})} & \geq\left\|P_{\varphi}^{g} f_{t(\varphi(z) /|\varphi(z)|)}\right\|_{H_{\beta}^{\infty}} \asymp \sup _{w \in \mathbb{B}}(1-|w|)^{\beta+1}\left|g C_{\varphi} f_{t(\varphi(z) /|\varphi(z)|)}(w)\right| \\
& \geq(1-|z|)^{\beta+1}|g(z)|\left|f_{t(\varphi(z) /|\varphi(z)|)}(\varphi(z))\right| \\
& =\frac{(1-|z|)^{\beta+1}|g(z)|}{(1-t|\varphi(z)|)^{\alpha}}
\end{aligned}
$$

for all $t \in(0,1)$. Letting $t \rightarrow 1^{-}$in (4.8), we have

$$
\left\|P_{\varphi}^{g}\right\|_{H_{\alpha, 0}^{\infty}(\mathbb{B}) \rightarrow H_{\beta}^{\infty}(\mathbb{B})} \geq C \frac{(1-|z|)^{\beta+1}|g(z)|}{(1-|\varphi(z)|)^{\alpha}} .
$$

For the constant function $1 \in H_{\alpha, 0}^{\infty}(\mathbb{B})$ we obtain

$$
\left\|P_{\varphi}^{g} 1\right\|_{H_{\beta}^{q}} \asymp \sup _{w \in \mathbb{B}}(1-|w|)^{\beta+1}\left|g C_{\varphi} 1(w)\right| \geq(1-|z|)^{\beta+1}|g(z)| .
$$

Inequality (4.10) shows that the estimate in (4.9) also holds when $\varphi(z)=0$.

Hence, from (4.9) we obtain

$$
\left\|P_{\varphi}^{g}\right\|_{H_{\alpha, 0}^{\infty}(\mathbb{B}) \rightarrow H_{\beta}^{\infty}(\mathbb{B})} \geq \operatorname{Csup}_{z \in \mathbb{B}} \frac{(1-|z|)^{\beta+1}|g(z)|}{(1-|\varphi(z)|)^{\alpha}},
$$

which along with the obvious inequality

$$
\left\|P_{\varphi}^{g}\right\|_{H_{\alpha}^{\infty}(\mathbb{B}) \rightarrow H_{\beta}^{\infty}(\mathbb{B})} \geq\left\|P_{\varphi}^{g}\right\|_{H_{\alpha, 0}^{\infty}(\mathbb{B}) \rightarrow H_{\beta}^{\infty}(\mathbb{B})}
$$

completes the proof of the theorem.

For the compactness of $P_{\varphi}^{g}: H_{\alpha}^{\infty}(\mathbb{B}) \quad\left(\right.$ or $\left.H_{\alpha, 0}^{\infty}(\mathbb{B})\right) \rightarrow H_{\beta}^{\infty}(\mathbb{B})$, we can also prove the following proposition which is similar to Proposition 2.6. 
Proposition 4.2. Let $\alpha, \beta>0$. Suppose that $g \in H(\mathbb{B})$ with $g(0)=0$ and $\varphi$ is a holomorphic self-map of $\mathbb{B}$ which induce the bounded operator $P_{\varphi}^{g}: H_{\alpha}^{\infty}(\mathbb{B}) \quad\left(\right.$ or $\left.H_{\alpha, 0}^{\infty}(\mathbb{B})\right) \rightarrow H_{\beta}^{\infty}(\mathbb{B})$. Then $P_{\varphi}^{g}: H_{\alpha}^{\infty}(\mathbb{B}) \quad\left(\right.$ or $\left.H_{\alpha, 0}^{\infty}(\mathbb{B})\right) \rightarrow H_{\beta}^{\infty}(\mathbb{B})$ is compact if and only if for every bounded sequence $\left\{f_{j}\right\}_{j \in \mathbb{N}}$ in $H_{\alpha}^{\infty}(\mathbb{B}) \quad\left(\right.$ or $\left.H_{\alpha, 0}^{\infty}(\mathbb{B})\right)$ which converges to 0 uniformly on compact subsets of $\mathbb{B},\left\{P_{\varphi}^{g} f_{j}\right\}_{j \in \mathbb{N}}$ converges to 0 in $H_{\beta}^{\infty}(\mathbb{B})$.

Theorem 4.3. Let $\alpha, \beta>0$. Suppose that $g \in H(\mathbb{B})$ with $g(0)=0$ and $\varphi$ is a holomorphic selfmap of $\mathbb{B}$ such that $P_{\varphi}^{g}: H_{\alpha}^{\infty}(\mathbb{B})\left(\right.$ or $\left.H_{\alpha, 0}^{\infty}(\mathbb{B})\right) \rightarrow H_{\beta}^{\infty}(\mathbb{B})$ is bounded. Then the essential norm $\left\|P_{\varphi}^{g}\right\|_{e, H_{\alpha}^{\infty}(\mathbb{B})\left(\text { or } H_{\alpha, 0}^{\infty}(\mathbb{B})\right) \rightarrow H_{\beta}^{\infty}(\mathbb{B})}$ is comparable to

$$
\limsup _{|\varphi(z)| \rightarrow 1^{-}} \frac{(1-|z|)^{\beta+1}|g(z)|}{(1-|\varphi(z)|)^{\alpha}} .
$$

In particular, $P_{\varphi}^{g}: H_{\alpha}^{\infty}(\mathbb{B})\left(\right.$ or $\left.H_{\alpha, 0}^{\infty}(\mathbb{B})\right) \rightarrow H_{\beta}^{\infty}(\mathbb{B})$ is compact if and only if

$$
\lim _{|\varphi(z)| \rightarrow 1^{-}} \frac{(1-|z|)^{\beta+1}|g(z)|}{(1-|\varphi(z)|)^{\alpha}}=0 .
$$

Proof. First we consider the family $\left\{f_{w}\right\}_{w \in \mathbb{B}}$ where

$$
f_{w}(z)=\frac{1-|w|}{(1-\langle z, w\rangle)^{\alpha+1}}
$$

We can easily check that $f_{w} \in H_{\alpha, 0}^{\infty}(\mathbb{B}),\left\|f_{w}\right\|_{H_{\alpha}^{\infty}} \leq 1$ for all $w \in \mathbb{B}$ and $f_{w} \rightarrow 0$ uniformly on compact subsets of $\mathbb{B}$ as $|w| \rightarrow 1^{-}$. Hence [40, page 296, Theorem 2] implies that $f_{w} \rightarrow 0$ weakly in $H_{\alpha, 0}^{\infty}(\mathbb{B})$ as $|w| \rightarrow 1^{-}$.

If $\|\varphi\|_{\infty}<1$, then as in the proof of [26, Theorem 3] it can be seen that the operator $P_{\varphi}^{g}: H_{\alpha}^{\infty}(\mathbb{B}) \quad\left(\right.$ or $\left.H_{\alpha, 0}^{\infty}(\mathbb{B})\right) \rightarrow H_{\beta}^{\infty}(\mathbb{B})$ is compact, so that

$$
\left\|P_{\varphi}^{g}\right\|_{e, H_{\alpha}^{\infty}(\mathbb{B})}\left(\text { or } H_{\alpha, 0}^{\infty}(\mathbb{B})\right) \rightarrow H_{\beta}^{\infty}(\mathbb{B})=0
$$

On the other hand, the limit in (4.13) is vacuously equal to zero, from which the result follows in this case. If $\|\varphi\|_{\infty}=1$, then take a sequence $\left\{\varphi\left(z_{j}\right)\right\}_{j \in \mathbb{N}}$ in $\mathbb{B}$ with $\left|\varphi\left(z_{j}\right)\right| \rightarrow 1$ as $j \rightarrow \infty$ and put $F_{j}(z)=f_{\varphi\left(z_{j}\right)}(z)$ for each $j \in \mathbb{N}$. Then $\left\{F_{j}\right\}_{j \in \mathbb{N}}$ is a bounded sequence in $H_{\alpha, 0}^{\infty}(\mathbb{B})$ 
and $\left\{F_{j}\right\}_{j \in \mathbb{N}}$ converges to 0 weakly in $H_{\alpha, 0}^{\infty}(\mathbb{B})$, as $j \rightarrow \infty$. Hence for every compact operator $\mathcal{K}: H_{\alpha, 0}^{\infty}(\mathbb{B}) \rightarrow H_{\beta}^{\infty}(\mathbb{B})$ we have $\left\|\mathcal{K} F_{j}\right\|_{H_{\beta}^{\infty}} \rightarrow 0$ as $j \rightarrow \infty$. So we have

$$
\begin{aligned}
\left\|P_{\varphi}^{g}-\mathcal{K}\right\|_{H_{\alpha, 0}^{\infty}(\mathbb{B}) \rightarrow H_{\beta}^{\infty}(\mathbb{B})} & \geq \limsup _{j \rightarrow \infty}\left\|P_{\varphi}^{g} F_{j}-\mathcal{K} F_{j}\right\|_{H_{\beta}^{\infty}} \\
& \geq \limsup _{j \rightarrow \infty}\left\|P_{\varphi}^{g} F_{j}\right\|_{H_{\beta}^{\infty}} \\
& \asymp \limsup _{j \rightarrow \infty} \sup _{w \in \mathbb{B}}(1-|w|)^{\beta+1}|g(w)|\left|F_{j}(\varphi(w))\right| \\
& \geq \limsup _{j \rightarrow \infty}\left(1-\left|z_{j}\right|\right)^{\beta+1}\left|g\left(z_{j}\right)\right|\left|F_{j}\left(\varphi\left(z_{j}\right)\right)\right| \\
& \geq \frac{1}{2^{\alpha+1}} \limsup _{j \rightarrow \infty} \frac{\left(1-\left|z_{j}\right|\right)^{\beta+1}\left|g\left(z_{j}\right)\right|}{\left(1-\left|\varphi\left(z_{j}\right)\right|\right)^{\alpha}},
\end{aligned}
$$

for all compact operators $\mathcal{K}: H_{\alpha, 0}^{\infty}(\mathbb{B}) \rightarrow H_{\beta}^{\infty}(\mathbb{B})$. Taking the infimum over the set of all compact operators $\mathcal{K}: H_{\alpha, 0}^{\infty}(\mathbb{B}) \rightarrow H_{\beta}^{\infty}(\mathbb{B})$, we obtain

$$
\left\|P_{\varphi}^{g}\right\|_{e, H_{\alpha, 0}^{\infty}(\mathbb{B}) \rightarrow H_{\beta}^{\infty}(\mathbb{B})} \geq C \limsup _{j \rightarrow \infty} \frac{\left(1-\left|z_{j}\right|\right)^{\beta+1}\left|g\left(z_{j}\right)\right|}{\left(1-\left|\varphi\left(z_{j}\right)\right|\right)^{\alpha}}
$$

Combining this with the estimate $\left\|P_{\varphi}^{g}\right\|_{e, H_{\alpha}^{\infty}(\mathbb{B}) \rightarrow H_{\beta}^{\infty}(\mathbb{B})} \geq\left\|P_{\varphi}^{g}\right\|_{e, H_{\alpha, 0}^{\infty}(\mathbb{B}) \rightarrow H_{\beta}^{\infty}(\mathbb{B})}$, we have

$$
\left\|P_{\varphi}^{g}\right\|_{e, H_{\alpha}^{\infty}(\mathbb{B}) \rightarrow H_{\beta}^{\infty}(\mathbb{B})} \geq C \limsup _{|\varphi(z)| \rightarrow 1^{-}} \frac{(1-|z|)^{\beta+1}|g(z)|}{(1-|\varphi(z)|)^{\alpha}}
$$

Next we prove an upper estimate. Assume that $\left\{r_{l}\right\}_{l \in \mathbb{N}} \subset(0,1)$ is a sequence which increasingly converges to 1 . For this $\left\{r_{l}\right\}_{l \in \mathbb{N}}$, we define the operators defined by

$$
P_{r_{l} \varphi}^{g} f(z)=\int_{0}^{1} g(t z) f\left(r_{l} \varphi(t z)\right) \frac{d t}{t}
$$

As in the proof of $\left[26\right.$, Theorem 3], Proposition 4.2 shows that $P_{r_{l} \varphi}^{g}: H_{\alpha}^{\infty}(\mathbb{B}) \rightarrow H_{\beta}^{\infty}(\mathbb{B})$ is compact for each $l \in \mathbb{N}$.

Put

$$
M_{2}:=\limsup _{|\varphi(z)| \rightarrow 1^{-}} \frac{(1-|z|)^{\beta+1}|g(z)|}{(1-|\varphi(z)|)^{\alpha}}
$$


and fix $\varepsilon>0$. Then we can choose $R \in(0,1)$ such that

$$
\frac{(1-|z|)^{\beta+1}|g(z)|}{(1-|\varphi(z)|)^{\alpha}}<M_{2}+\varepsilon
$$

if $R<|\varphi(z)|<1$. Take $f \in H_{\alpha}^{\infty}(\mathbb{B})$ with $\|f\|_{H_{\alpha}^{\infty}} \leq 1$ and an integer $l \in \mathbb{N}$. By Lemma 3.1 and Lemma 3.2, we have

$$
\begin{aligned}
\left\|P_{\varphi}^{g} f-P_{r_{l} \varphi}^{g} f\right\|_{H_{\beta}^{\infty}} \asymp & \sup _{z \in \mathbb{B}}(1-|z|)^{\beta+1}\left|\Re\left[P_{\varphi}^{g} f\right](z)-\Re\left[P_{r_{l} \varphi}^{g} f\right](z)\right| \\
= & \sup _{z \in \mathbb{B}}(1-|z|)^{\beta+1}|g(z)|\left|f(\varphi(z))-f\left(r_{l} \varphi(z)\right)\right| \\
= & \sup _{|\varphi(z)| \leq R}(1-|z|)^{\beta+1}|g(z)|\left|f(\varphi(z))-f\left(r_{l} \varphi(z)\right)\right| \\
& +\sup _{R<|\varphi(z)|<1}(1-|z|)^{\beta+1}|g(z)|\left|f(\varphi(z))-f\left(r_{l} \varphi(z)\right)\right| .
\end{aligned}
$$

By using the mean value theorem and the asymptotic relation

$$
\sup _{z \in \mathbb{B}}(1-|z|)^{\alpha+1}|\nabla f(z)| \asymp \sup _{z \in \mathbb{B}}(1-|z|)^{\alpha+1}|\Re f(z)|,
$$

we obtain

$$
\begin{aligned}
\sup _{|\varphi(z)| \leq R}\left|f(\varphi(z))-f\left(r_{l} \varphi(z)\right)\right| & \leq \sup _{|\varphi(z)| \leq R}\left(1-r_{l}\right)|\varphi(z)| \sup _{|w| \leq R}|\nabla f(w)| \\
& \leq \frac{\left(1-r_{l}\right) R}{(1-R)^{\alpha+1}} \sup _{w \in \mathbb{B}}(1-|w|)^{\alpha+1}|\nabla f(w)| \\
& \asymp \frac{\left(1-r_{l}\right) R}{(1-R)^{\alpha+1}} \sup _{w \in \mathbb{B}}(1-|w|)^{\alpha+1}|\Re f(w)| \\
& \asymp \frac{\left(1-r_{l}\right) R}{(1-R)^{\alpha+1}}\|f\|_{H_{\alpha}^{\infty}}
\end{aligned}
$$

Since the boundedness of $P_{\varphi}^{g}: H_{\alpha}^{\infty}(\mathbb{B}) \rightarrow H_{\beta}^{\infty}(\mathbb{B})$ implies that $P_{\varphi}^{g} 1 \in H_{\beta}^{\infty}(\mathbb{B})$, we see

$$
\sup _{z \in \mathbb{B}}(1-|z|)^{\beta+1}|g(z)|<\infty
$$

and so we have

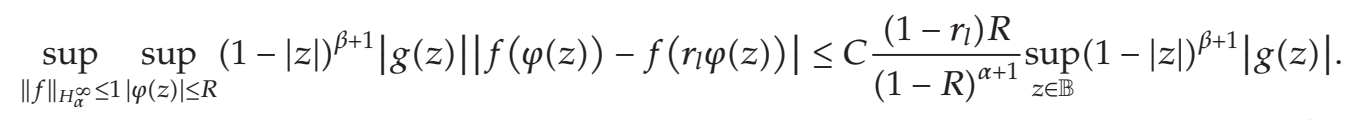


On the other hand, the monotonicity of $M_{\infty}(f, r)$ shows

$$
\left|f\left(r_{l} \varphi(z)\right)\right| \leq(1-|\varphi(z)|)^{-\alpha}\left\|f_{r_{l}}\right\|_{H_{\alpha}^{\infty}} \leq(1-|\varphi(z)|)^{-\alpha}\|f\|_{H_{\alpha}^{\infty}}
$$

Thus we have

$$
\begin{aligned}
\sup _{\|f\|_{H_{\alpha}^{\infty} \leq 1}} \sup _{R<|\varphi(z)|<1}(1-|z|)^{\beta+1}|g(z)|\left|f(\varphi(z))-f\left(r_{l} \varphi(z)\right)\right| & \leq 2 \sup _{R<|\varphi(z)|<1} \frac{(1-|z|)^{\beta+1}|g(z)|}{(1-|\varphi(z)|)^{\alpha}} \\
& \leq 2\left(M_{2}+\varepsilon\right) .
\end{aligned}
$$

From (4.23), (4.27), (4.29), and the compactness of $P_{r_{l} \varphi}^{g}$, we obtain

$$
\begin{aligned}
\left\|P_{\varphi}^{g}\right\|_{e, H_{\alpha}^{\infty}(\mathbb{B}) \rightarrow H_{\beta}^{\infty}(\mathbb{B})} & \leq\left\|P_{\varphi}^{g}-P_{r_{l} \varphi}^{g}\right\|_{H_{\alpha}^{\infty}(\mathbb{B}) \rightarrow H_{\beta}^{\infty}(\mathbb{B})} \\
& \leq C \frac{\left(1-r_{l}\right) R}{(1-R)^{\alpha+1}} \sup _{z \in \mathbb{B}}(1-|z|)^{\beta+1}|g(z)|+2\left(M_{2}+\varepsilon\right) .
\end{aligned}
$$

Letting $l \rightarrow \infty$ and $\varepsilon \rightarrow 0$, we have

$$
\left\|P_{\varphi}^{g}\right\|_{e, H_{\alpha}^{\infty}(\mathbb{B}) \rightarrow H_{\beta}^{\infty}(\mathbb{B})} \leq 2 \limsup _{|\varphi(z)| \rightarrow 1^{-}} \frac{(1-|z|)^{\beta+1}|g(z)|}{(1-|\varphi(z)|)^{\alpha}}
$$

This completes the proof.

When $P_{\varphi}^{g}: H_{\alpha}^{\infty}(\mathbb{B}) \quad\left(\right.$ or $\left.H_{\alpha, 0}^{\infty}(\mathbb{B})\right) \rightarrow H_{\beta, 0}^{\infty}(\mathbb{B})$ is bounded, we see that $g \in H_{\beta+1,0}^{\infty}(\mathbb{B})$. By a standard argument as in the proof of [26, Corollary 3], we have

$$
\limsup _{|z| \rightarrow 1^{-}} \frac{(1-|z|)^{\beta+1}|g(z)|}{(1-|\varphi(z)|)^{\alpha}}=\limsup _{|\varphi(z)| \rightarrow 1^{-}} \frac{(1-|z|)^{\beta+1}|g(z)|}{(1-|\varphi(z)|)^{\alpha}}
$$

and so

$$
\left\|P_{\varphi}^{g}\right\|_{e, H_{\alpha}^{\infty}(\mathbb{B})\left(\text { or } H_{\alpha, 0}^{\infty}(\mathbb{B})\right) \rightarrow H_{\beta, 0}^{\infty}(\mathbb{B})} \asymp \limsup _{|z| \rightarrow 1^{-}} \frac{(1-|z|)^{\beta+1}|g(z)|}{(1-|\varphi(z)|)^{\alpha}} .
$$

Hence we obtain the following characterization for the compactness of the operator $P_{\varphi}^{g}$ : $H_{\alpha}^{\infty}(\mathbb{B}) \quad\left(\right.$ or $\left.H_{\alpha, 0}^{\infty}(\mathbb{B})\right) \rightarrow H_{\beta, 0}^{\infty}(\mathbb{B})$. 
Corollary 4.4. Let $\alpha, \beta>0$. Suppose that $g \in H(\mathbb{B})$ with $g(0)=0$ and $\varphi$ is a holomorphic self-map of $\mathbb{B}$ such that $P_{\varphi}^{g}: H_{\alpha}^{\infty}(\mathbb{B})\left(\right.$ or $\left.H_{\alpha, 0}^{\infty}(\mathbb{B})\right) \rightarrow H_{\beta, 0}^{\infty}(\mathbb{B})$ is bounded. Then $P_{\varphi}^{g}: H_{\alpha}^{\infty}(\mathbb{B}) \quad\left(\right.$ or $\left.H_{\alpha, 0}^{\infty}(\mathbb{B})\right) \rightarrow$ $H_{\beta, 0}^{\infty}(\mathbb{B})$ is compact if and only if

$$
\lim _{|z| \rightarrow 1^{-}} \frac{(1-|z|)^{\beta+1}|g(z)|}{(1-|\varphi(z)|)^{\alpha}}=0 .
$$

\section{Acknowledgment}

The second author of this paper is supported by Grant-in-Aid for Young Scientists (Start-up; no.20840004).

\section{References}

[1] S. Stević, "On Bloch-type functions with Hadamard gaps," Abstract and Applied Analysis, vol. 2007, Article ID 39176, 8 pages, 2007.

[2] S. Li and S. Stević, "Weighted-Hardy functions with Hadamard gaps on the unit ball," Applied Mathematics and Computation, vol. 212, no. 1, pp. 229-233, 2009.

[3] K. L. Avetisyan, "Hardy-Bloch type spaces and lacunary series on the polydisk," Glasgow Mathematical Journal, vol. 49, no. 2, pp. 345-356, 2007.

[4] C. C. Cowen and B. D. MacCluer, Composition Operators on Spaces of Analytic Functions, Studies in Advanced Mathematics, CRC Press, Boca Raton, Fla, USA, 1995.

[5] S. Li and S. Stević, "Weighted composition operators from $\alpha$-Bloch space to $H^{\infty}$ on the polydisc," Numerical Functional Analysis and Optimization, vol. 28, no. 7-8, pp. 911-925, 2007.

[6] S. Li and S. Stević, "Weighted composition operators between $H^{\infty}$ and $\alpha$-Bloch spaces in the unit ball," Taiwanese Journal of Mathematics, vol. 12, no. 7, pp. 1625-1639, 2008.

[7] S. Stević, "Weighted composition operators between mixed norm spaces and $H_{\alpha}^{\infty}$ spaces in the unit ball," Journal of Inequalities and Applications, vol. 2007, Article ID 28629, 9 pages, 2007.

[8] S. Stević, "Norm of weighted composition operators from Bloch space to $H_{\mu}^{\infty}$ on the unit ball," Ars Combinatoria, vol. 88, pp. 125-127, 2008.

[9] S. Stević, "Essential norms of weighted composition operators from the $\alpha$-Bloch space to a weightedtype space on the unit ball," Abstract and Applied Analysis, vol. 2008, Article ID 279691, 11 pages, 2008.

[10] S. Stević, "Weighted composition operators from weighted Bergman spaces to weighted-type spaces on the unit ball," Applied Mathematics and Computation, vol. 212, no. 2, pp. 499-504, 2009.

[11] S.-I. Ueki, "Composition operators on the Privalov spaces of the unit ball of $\mathbb{C}^{n}$," Journal of the Korean Mathematical Society, vol. 42, no. 1, pp. 111-127, 2005.

[12] S.-I. Ueki, "Weighted composition operators between weighted Bergman spaces in the unit ball of $\mathbb{C}^{n}$, " Nihonkai Mathematical Journal, vol. 16, no. 1, pp. 31-48, 2005.

[13] S.-I. Ueki and L. Luo, "Essential norms of weighted composition operators between weighted Bergman spaces of the ball," Acta Scientiarum Mathematicarum, vol. 74, no. 3-4, pp. 829-843, 2008.

[14] E. Wolf, "Weighted composition operators between weighted Bergman spaces and weighted Banach spaces of holomorphic functions," Revista Matemática Complutense, vol. 21, no. 2, pp. 475-480, 2008.

[15] H.-M. Xu and T.-S. Liu, "Weighted composition operators between Hardy spaces on the unit ball," Chinese Quarterly Journal of Mathematics, vol. 19, no. 2, pp. 111-119, 2004.

[16] W. Yang, "Weighted composition operators from Bloch-type spaces to weighted-type spaces," to appear in Ars Combinatoria.

[17] X. Zhu, "Weighted composition operators from $F(p, q, s)$ spaces to $H_{\mu}^{\infty}$ spaces," Abstract and Applied Analysis, vol. 2009, Article ID 290978, 14 pages, 2009.

[18] S. Li and S. Stević, "Products of composition and integral type operators from $H^{\infty}$ to the Bloch space," Complex Variables and Elliptic Equations, vol. 53, no. 5, pp. 463-474, 2008.

[19] S. Li and S. Stević, "Products of Volterra type operator and composition operator from $H^{\infty}$ and Bloch spaces to Zygmund spaces," Journal of Mathematical Analysis and Applications, vol. 345, no. 1, pp. 40-52, 2008. 
[20] S. Li and S. Stević, "Products of integral-type operators and composition operators between Blochtype spaces," Journal of Mathematical Analysis and Applications, vol. 349, no. 2, pp. 596-610, 2009.

[21] S. Stević, "Products of integral-type operators and composition operators from the mixed norm space to Bloch-type spaces," Siberian Mathematical Journal, vol. 50, no. 4, pp. 726-736, 2009.

[22] S. Li and S. Stević, "Generalized composition operators on Zygmund spaces and Bloch type spaces," Journal of Mathematical Analysis and Applications, vol. 338, no. 2, pp. 1282-1295, 2008.

[23] S. Stević, "Generalized composition operators from logarithmic Bloch spaces to mixed-norm spaces," Utilitas Mathematica, vol. 77, pp. 167-172, 2008.

[24] S. Stević, "On a new operator from $H^{\infty}$ to the Bloch-type space on the unit ball," Utilitas Mathematica, vol. 77, pp. 257-263, 2008.

[25] S. Stević, "On a new integral-type operator from the weighted Bergman space to the Bloch-type space on the unit ball," Discrete Dynamics in Nature and Society, vol. 2008, Article ID 154263, 14 pages, 2008.

[26] S. Stević, "On a new integral-type operator from the Bloch space to Bloch-type spaces on the unit ball," Journal of Mathematical Analysis and Applications, vol. 354, no. 2, pp. 426-434, 2009.

[27] Z. Hu, "Extended Cesàro operators on mixed norm spaces," Proceedings of the American Mathematical Society, vol. 131, no. 7, pp. 2171-2179, 2003.

[28] S. Li and S. Stević, "Compactness of Riemann-Stieltjes operators between $F(p, q, s)$ spaces and $\alpha$-Bloch spaces," Publicationes Mathematicae Debrecen, vol. 72, no. 1-2, pp. 111-128, 2008.

[29] S. Li and S. Stević, "Riemann-Stieltjes operators between different weighted Bergman spaces," Bulletin of the Belgian Mathematical Society. Simon Stevin, vol. 15, no. 4, pp. 677-686, 2008.

[30] S. Li and S. Stević, "Riemann-Stieltjes operators between mixed norm spaces," Indian Journal of Mathematics, vol. 50, no. 1, pp. 177-188, 2008.

[31] S. Stević, "On an integral operator on the unit ball in $\mathbb{C}^{n}$, " Journal of Inequalities and Applications, vol. 2005, no. 1, pp. 81-88, 2005.

[32] S. Stević, "Boundedness and compactness of an integral operator on a weighted space on the polydisc," Indian Journal of Pure and Applied Mathematics, vol. 37, no. 6, pp. 343-355, 2006.

[33] S. Stević, "Boundedness and compactness of an integral operator in a mixed norm space on the polydisk," Sibirskiŭ Matematicheskiŭ Zhurnal, vol. 48, no. 3, pp. 694-706, 2007.

[34] S.-I. Ueki and L. Luo, "Compact weighted composition operators and multiplication operators between Hardy spaces," Abstract and Applied Analysis, vol. 2008, Article ID 196498, 12 pages, 2008.

[35] L. Luo and J. H. Shi, "Composition operators between Hardy spaces on the unit ball," Acta Mathematica Sinica, vol. 44, no. 2, pp. 209-216, 2001 (Chinese).

[36] B. D. MacCluer, "Compact composition operators on $H^{p}\left(B_{N}\right)$," The Michigan Mathematical Journal, vol. 32, no. 2, pp. 237-248, 1985.

[37] Ž. Čučković and R. Zhao, "Weighted composition operators between different weighted Bergman spaces and different Hardy spaces," Illinois Journal of Mathematics, vol. 51, no. 2, pp. 479-498, 2007.

[38] S. C. Power, "Hörmander's Carleson theorem for the ball," Glasgow Mathematical Journal, vol. 26, no. 1, pp. 13-17, 1985.

[39] S. Stević, "Composition operators between $H^{\infty}$ and $\alpha$-Bloch spaces on the polydisc," Zeitschrift für Analysis und ihre Anwendungen, vol. 25, no. 4, pp. 457-466, 2006.

[40] A. L. Shields and D. L. Williams, "Bonded projections, duality, and multipliers in spaces of analytic functions," Transactions of the American Mathematical Society, vol. 162, pp. 287-302, 1971. 


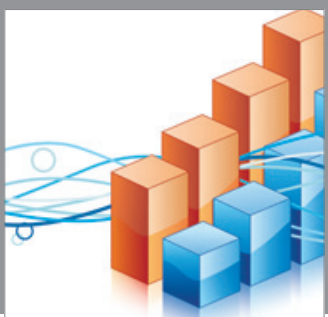

Advances in

Operations Research

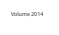

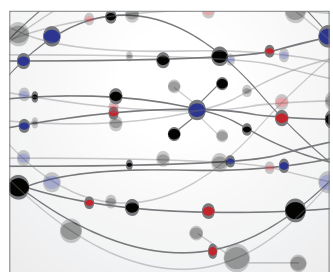

\section{The Scientific} World Journal
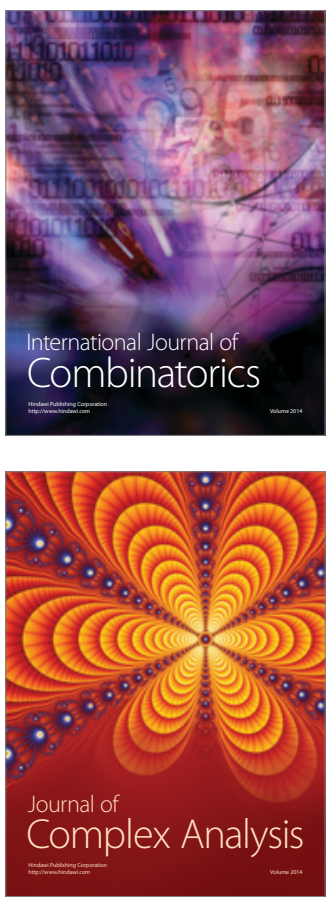

International Journal of

Mathematics and

Mathematical

Sciences
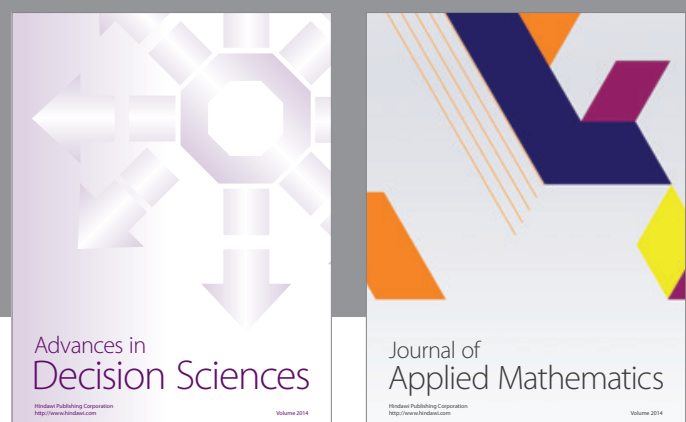

Journal of

Applied Mathematics
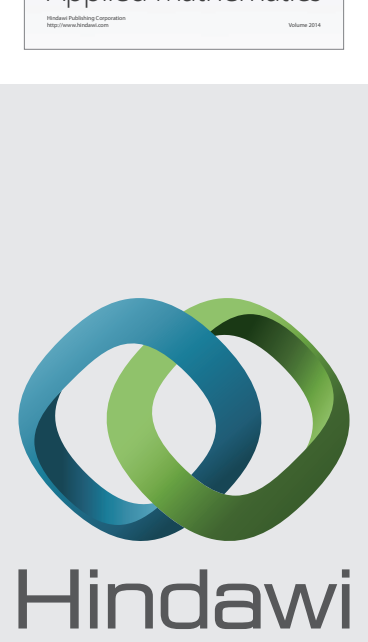

Submit your manuscripts at http://www.hindawi.com
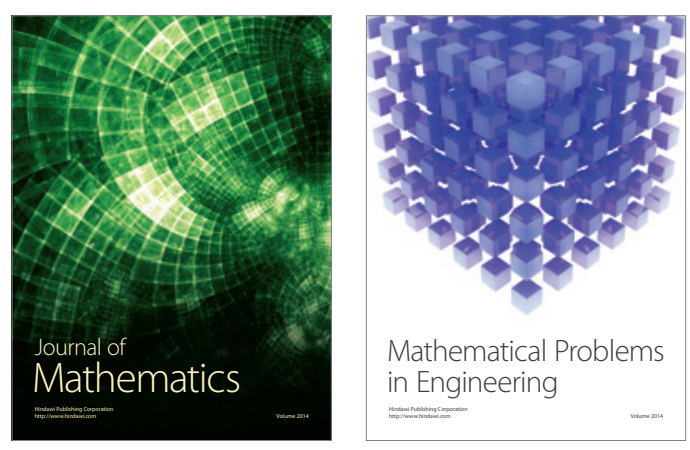

Mathematical Problems in Engineering
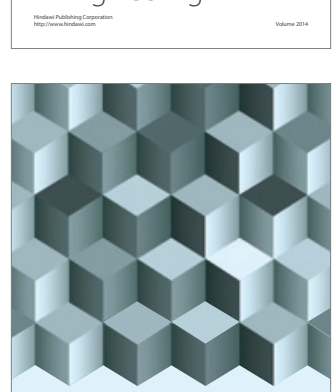

Journal of

Function Spaces
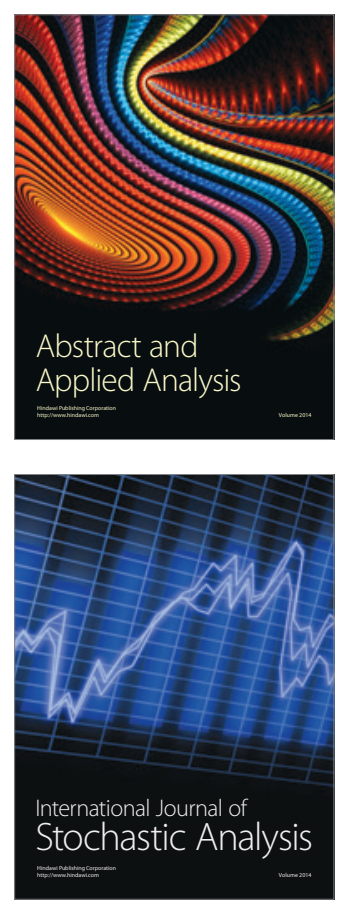

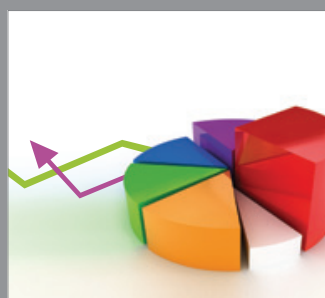

ournal of

Probability and Statistics

Promensencen
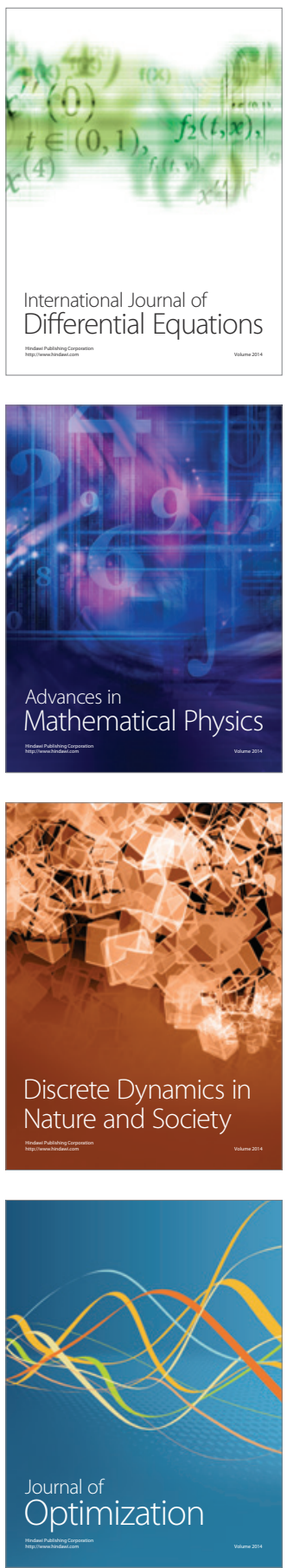